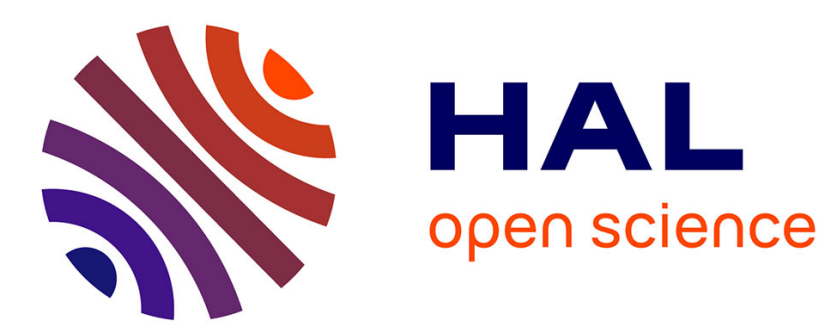

\title{
Multimodal electromechanical model of piezoelectric transformers by Hamilton's principle
}

Clément Nadal, François Pigache

\section{To cite this version:}

Clément Nadal, François Pigache. Multimodal electromechanical model of piezoelectric transformers by Hamilton's principle. IEEE Transactions on Ultrasonics, Ferroelectrics and Frequency Control, 2009, vol. 56, pp. 2530-2543. 10.1109/TUFFC.2009.1340 . hal-00780461

\section{HAL Id: hal-00780461 \\ https://hal.science/hal-00780461}

Submitted on 24 Jan 2013

HAL is a multi-disciplinary open access archive for the deposit and dissemination of scientific research documents, whether they are published or not. The documents may come from teaching and research institutions in France or abroad, or from public or private research centers.
L'archive ouverte pluridisciplinaire HAL, est destinée au dépôt et à la diffusion de documents scientifiques de niveau recherche, publiés ou non, émanant des établissements d'enseignement et de recherche français ou étrangers, des laboratoires publics ou privés. 


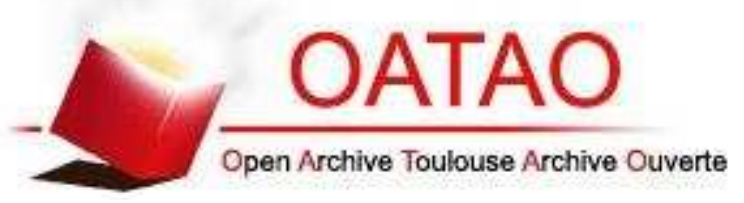

Open Archive Toulouse Archive Ouverte (OATAO)

OATAO is an open access repository that collects the work of Toulouse researchers and makes it freely available over the web where possible.

This is an author-deposited version published in: http://oatao.univ-toulouse.fr/ Eprints ID: 8050

To link to this article: DOI:10.1109/TUFFC.2009.1340

http://dx.doi.org/10.1109/TUFFC.2009.1340

\section{To cite this version:}

Nadal, Clément and Pigache, François Multimodal electromechanical model of piezoelectric transformers by Hamilton's principle. (2009) IEEE Transactions on Ultrasonics, Ferroelectrics and Frequency Control, vol. 56 ( $\left.\mathrm{n}^{\circ} 11\right)$. pp. 25302543. ISSN 0885-3010

Any correspondence concerning this service should be sent to the repository administrator: staff-oatao@inp-toulouse.fr 


\title{
Multimodal Electromechanical Model of Piezoelectric Transformers by Hamilton's Principle
}

\author{
Clement Nadal and Francois Pigache
}

\begin{abstract}
This work deals with a general energetic approach to establish an accurate electromechanical model of a piezoelectric transformer (PT). Hamilton's principle is used to obtain the equations of motion for free vibrations. The modal characteristics (mass, stiffness, primary and secondary electromechanical conversion factors) are also deduced. Then, to illustrate this general electromechanical method, the variational principle is applied to both homogeneous and nonhomogeneous Rosen-type PT models. A comparison of modal parameters, mechanical displacements, and electrical potentials are presented for both models. Finally, the validity of the electrodynamical model of nonhomogeneous Rosen-type PT is confirmed by a numerical comparison based on a finite elements method and an experimental identification.
\end{abstract}

\section{INTRODUCTION}

$\mathrm{T}$ HE emergence of piezoelectric transformers coincides with the development in the 1950s of ferroelectric ceramics belonging to the perovskites crystalline family. The first architecture was proposed by Rosen [1], who designed a step-up piezoelectric transformer (PT) made in barium titanate $\left(\mathrm{BaTiO}_{3}\right)$ rod in 1956. In addition to providing small size and weight, PTs offer outstanding performance in terms of galvanic insulation, voltage ratio, and efficiency. Furthermore, compared with conventional electromagnetic transformers, PTs are free from electromagnetic interference. They are consequently more suitable for low-power applications, high efficiency, and small embedded systems.

For some years, a wide range of piezoelectric transformer structures has been developed to ensure various electrical requirements, also considering fixed constraints or environmental conditions. Substantial performance improvements have been reached with the development of new piezoactive materials and manufacturing process.

Typically, PTs provide optimal performances when they are supplied by voltage power supply close to one of their mechanical resonance frequencies. Furthermore, these frequencies depend strongly on dimensions, shape, and mechanical properties of the medium. This implies that vibratory eigenmodes must be clearly defined and

The authors are with the Universite de Toulouse; INP, UPS; Laboratoire Plasma et Conversion d'Energie (LAPLACE); Ecole Nationale Supérieure d'Electrotechnique, d'Electronique, d’Informatique, d'Hydraulique et des Télécommunications (ENSEEIHT), F-31071 Toulouse cedex 7, France, and CNRS; LAPLACE; F-31071 Toulouse, France.

Digital Object Identifier 10.1109/TUFFC.2009.1340 convenient models have to describe the electromechanical behavior. The development of analytical models [2]-[3] is particularly suitable for implementation into optimization algorithms [4] to deduce optimal dimensions.

The present paper relies on the development of a general analytical method to treat the modeling of any PT for several vibratory modes. With this analytical multimodal approach, the performance can also be predicted for different kinds of PT design.

First, the general method based on Hamilton's principle and matrix formulation is detailed. Then, this method is illustrated by modeling a classical Rosen-type transformer with consideration of nonhomogenous electromechanical properties along the displacement axis. The same method is also used for this transformer considering a current approximation of a homogenous elastic medium. As result, both problem formulations are balanced and thus highlight the influence of this assumption in terms of precision.

Finally, the modal parameters (i.e., the modal mass, the modal stiffness, the transformer ratio, the resonant frequency at shorted circuit) will be extracted and compared with those that come from a numerical study using a finite elements method and an experimental identification.

\section{General Analytical Formulation}

Following the example of conventional magnetic transformers, the piezoelectric transformers are composed of primary and secondary sections with the difference that the coupling lies in an electromechanical conversion. The transformer is driven by a sinusoidal voltage power supply. The frequency of applied voltage usually coincides with a fundamental mode of vibration to take advantage of the best electrical performances of the PT (efficiency, voltage step-up ratio).

To establish a multimodal model of any piezoelectric transformer, a general analytical formulation is presented in following sections. First, the Hamilton's principle is detailed, governed by matrix formulation. Then, a methodology to deduce modal shapes is undertaken to get to the electromechanical model.

\section{A. Electromechanical Modeling}

To access the electromechanical behavior of the PT, an energy approach based on Hamilton's principle seems to 
be the most appropriate choice. From [5], a generalized form of Hamilton's principle for electromechanical systems is

$$
\delta \int_{t_{1}}^{t_{2}} L d t+\int_{t_{1}}^{t_{2}} \delta W d t=0
$$

where $L$ is the Lagrangian of the system and $W$ is the variational work done by the external forces. In the classical way, the Lagrangian of a piezoelectric structure is given by

$$
L=T-U+W_{e},
$$

where $T$ and $U$ are the kinetic and potential energies from which the modal mass and stiffness matrices are respectively deduced. A linear electrical energy term $W_{e}$, is added to take into account the electrical energy stored within the piezoelectric material. For a piezoelectric transformer of volume $\Omega$ and total surface $\Sigma$, the expression of kinetic energy simply takes the following form:

$$
T=\frac{1}{2} \int_{\Omega_{\text {in }}} \rho\{\dot{u}\}^{T}\{\dot{u}\} d \Omega+\frac{1}{2} \int_{\Omega_{\text {out }}} \rho\{\dot{u}\}^{T}\{\dot{u}\} d \Omega,
$$

where $\rho$ represents the material density; $\Omega_{\text {in }}$ and $\Omega_{\text {out }}$ are, respectively, the volumes of the driving and receiving parts. The kinetic energy is split into 2 contributions: that caused by the motion within the driving part and that caused by motion within the receiving part. The $3-\mathrm{D}$ vector $\{u\}$ is the displacement vector in the $x_{1}, x_{2}$, and $x_{3}$ directions. The superscript ${ }^{T}$ denotes the mathematical transpose.

The potential energy of a piezoelectric transformer is defined as

$$
U=\frac{1}{2} \int_{\Omega_{\mathrm{in}}}\{S\}^{T}\{T\} d \Omega+\frac{1}{2} \int_{\Omega_{\mathrm{out}}}\{S\}^{T}\{T\} d \Omega,
$$

where $\{S\}$ represents the strain vector and $\{T\}$ the stress vector. The electrical energy $W_{e}$ exists also within the whole piezoelectric transformer:

$$
W_{e}=\frac{1}{2} \int_{\Omega_{\mathrm{in}}}\{E\}^{T}\{D\} d \Omega+\frac{1}{2} \int_{\Omega_{\mathrm{out}}}\{E\}^{T}\{D\} d \Omega,
$$

where $\{D\}$ represents the electrical displacement vector and $\{E\}$ the electric field vector.

The variational work due to the external forces on the piezoelectric transformer is given by

$$
\delta W=\delta W_{\text {in }}+\delta W_{\text {out }},
$$

where $W_{\text {in }}$ and $W_{\text {out }}$ are, respectively, the applied electrical energies to the driving and receiving portions caused by the external charges. These variational electrical works can be expressed as

$$
\delta W_{\text {in }}=-\left\{q_{\text {in }}\right\}^{T}\left\{\delta v_{\text {in }}\right\} ; \quad \delta W_{\text {out }}=\left\{q_{\text {out }}\right\}^{T}\left\{\delta v_{\text {out }}\right\},
$$

where $\left\{q_{\text {in }}\right\}^{T},\left\{v_{\text {in }}\right\},\left\{q_{\text {out }}\right\}$ and $\left\{v_{\text {out }}\right\}$ are, respectively, the electrical charge and voltage vectors at the electrodes of the primary and secondary parts. The difference of sign between the primary and secondary external energies is due to the respective actuator and sensor behaviors of the driving and receiving sections.

To derive the potential and electrical energy expressions, the piezoelectric constitutive relationships relative to the primary and secondary sections will be properly included. Regarding the constitutive laws of the piezoelectric material, the driving part behaves like a piezoelectric actuator, which means that a mechanical deformation can be generated by applying an electrical field. The set of 2 independent variables $(S, E)$ is so chosen in the constitutive equations which take the following form:

$$
\begin{aligned}
& \{T\}=\left[c^{E}\right]\{S\}-[e]^{T}\{E\} \\
& \{D\}=[e]\{S\}+\left[\varepsilon^{S}\right]\{E\},
\end{aligned}
$$

where $\left[c^{E}\right],[e]$, and $\left[\varepsilon^{S}\right]$ are, respectively, the stiffness matrix (at constant electrical field), the piezoelectric constant matrix, and the dielectric permittivity matrix (at constant strain). The secondary part behaves like a sensor, which means that the mechanical strain and the electrical field can interact. The set of 2 independent variables $(S, D)$ is consequently selected to express the constitutive equations of the secondary part. They take the following form:

$$
\begin{aligned}
& \{T\}=\left[c^{D}\right]\{S\}-[h]^{T}\{D\} \\
& \{E\}=-[h]\{S\}+\left[\beta^{S}\right]\{D\},
\end{aligned}
$$

where $\left[c^{D}\right],[h]$, and $\left[\beta^{S}\right]$ are, respectively, the stiffness matrix (at constant electrical displacement), the piezoelectric constant matrix, and the dielectric impermittivity matrix (at constant strain).

Before the assumed modes are chosen, the strain-displacement relations must be developed according to the theory of linear elasticity. Within the framework of a modal study, the displacement vector can be defined in terms of several assumed modes and takes generally the following matrix formula [6]:

$$
\{u\}=\left[\begin{array}{c}
u_{1} \\
u_{2} \\
u_{3}
\end{array}\right]=\lambda_{m}\left(x_{1}, x_{2}, x_{3}\right)\left[\begin{array}{c}
\eta_{1}(t) \\
\vdots \\
\eta_{n}(t)
\end{array}\right] .
$$

This expression differentiates the geometrical domain from the time domain (harmonic vibrations assumption). In fact, the mechanical modal amplitude vector $\{\eta\}$ is only dependent on time and the assumed mechanical mode shape matrix $\lambda_{m}$ is only dependent on the coordinates $x_{1}, x_{1}$, and $x_{3}$. It must be emphasized that the solution remains general for $n$ assumed modes. 
The relationship between strain and displacement vectors can be obtained by applying the Green's relation in the case of a linear deformation assumption [7]. The strain vector is finally represented with the mechanical modal amplitude $\{\eta\}$ as shown:

$$
\{S\}=N_{m}\{\eta\} \text { where } N_{m}=L_{m} \lambda_{m},
$$

where $L_{m}$ is a $(6 \times 3)$-matrix differential operator depending on the coordinates system. As an example, in the Cartesian coordinates system, $L_{m}$ is

$$
L_{m}=\left(\begin{array}{cccccc}
\frac{\partial}{\partial x_{1}} & 0 & 0 & \frac{\partial}{\partial x_{2}} & 0 & \frac{\partial}{\partial x_{3}} \\
0 & \frac{\partial}{\partial x_{2}} & 0 & \frac{\partial}{\partial x_{1}} & \frac{\partial}{\partial x_{3}} & 0 \\
0 & 0 & \frac{\partial}{\partial x_{3}} & 0 & \frac{\partial}{\partial x_{2}} & \frac{\partial}{\partial x_{1}}
\end{array}\right)^{T} .
$$

Contrary to the strain vector, the assumed shapes on the electrical potential $\phi$ within the piezoelectric transformer have to be defined in the primary and secondary parts. The selected solution is to write the electrical potential in both sides as

$$
\begin{aligned}
& \phi=\lambda_{\text {in }}\left\{v_{\text {in }}\right\} \\
& \phi=\lambda_{\text {out }}\left\{v_{\text {out }}\right\},
\end{aligned}
$$

where $\lambda_{\text {in }}$ and $\lambda_{\text {out }}$ are the electrical mode shape matrix of the primary and secondary sides, respectively. Thereby, the electrical field in the piezoelectric transformer is given by

$$
\begin{aligned}
& \{E\}=L_{\text {in }} \phi \\
& \{E\}=L_{\text {out }} \phi,
\end{aligned}
$$

where the operator matrices $L_{\text {in }}$ and $L_{\text {out }}$ convert the assumed shape on the potential $\phi$ at the electrodes to an electrical field within the primary and secondary parts, respectively. As a consequence, the electrical field and the applied voltage relationships in both sections are

$$
\begin{aligned}
& \{E\}=N_{\text {in }}\left\{v_{\text {in }}\right\} \quad N_{\text {in }}=L_{\text {in }} \lambda_{\text {in }} \\
& \{E\}=N_{\text {out }}\left\{v_{\text {out }}\right\} \quad N_{\text {out }}=L_{\text {out }} \lambda_{\text {out }} .
\end{aligned}
$$

After the strain-displacement and the electrical field-voltage relations are defined, the equation of motion of the piezoelectric transformer can be derived by appropriately substituting $T, U$, and $W_{e}$ in (1), as described subsequently.

1) Kinetic Energy: By substituting (11) in (3) and rearranging the terms, the kinetic energy within transformer is

$$
T=\frac{1}{2}\{\dot{\eta}\}^{T}\left[M_{\text {in }}\right]\{\dot{\eta}\}+\frac{1}{2}\{\dot{\eta}\}^{T}\left[M_{\text {out }}\right]\{\dot{\eta}\},
$$

where $\left[M_{\mathrm{in}}\right]$ and $\left[M_{\text {out }}\right]$ are the modal mass matrix of the primary and secondary sections respectively, which are defined as

$$
\begin{aligned}
{\left[M_{\text {in }}\right] } & =\int_{\Omega_{\text {in }}} \lambda_{m}^{T} \rho \lambda_{m} d \Omega \\
{\left[M_{\text {out }}\right] } & =\int_{\Omega_{\text {out }}} \lambda_{m}^{T} \rho \lambda_{m} d \Omega
\end{aligned}
$$

2) Potential Energy: By substituting the expressions of the stress vector from the piezoelectric constitutive equations (8) and (9) in (4) and rearranging the terms, the potential energy of PT is the sum of both contributions of the driving and receiving parts given by

$$
\begin{aligned}
U_{\text {in }} & =\frac{1}{2}\{\eta\}^{T}\left[K_{\text {in }}\right]\{\eta\}-\frac{1}{2}\{\eta\}^{T}\left[\psi_{\text {in }}\right]\left\{v_{\text {in }}\right\} \\
U_{\text {out }} & =\frac{1}{2}\{\eta\}^{T}\left[K_{\text {out }}\right]\{\eta\}+\frac{1}{2}\{\eta\}^{T}\left[\psi_{\text {out }}\right]\left\{v_{\text {out }}\right\},
\end{aligned}
$$

where $\left[K_{\text {in }}\right]$ and $\left[K_{\text {out }}\right]$ are the modal stiffness matrix of the primary and secondary parts, respectively, which are defined as

$$
\begin{aligned}
{\left[K_{\text {in }}\right] } & =\int_{\Omega_{\text {in }}} N_{m}^{T}\left[c^{E}\right] N_{m} d \Omega \\
{\left[K_{\text {out }}\right] } & =\int_{\Omega_{\text {out }}} N_{m}^{T}\left(\left[c^{D}\right]-[h]^{T}\left[\beta^{S}\right]^{-1}[h]\right) N_{m} d \Omega,
\end{aligned}
$$

where $\left[\psi_{\text {in }}\right]$ and $\left[\psi_{\text {out }}\right]$ are the modal electromechanical coupling matrix parts which are expressed as

$$
\begin{aligned}
{\left[\psi_{\text {in }}\right] } & =\int_{\Omega_{\text {in }}} N_{m}^{T}[e]^{T} N_{\text {in }} d \Omega \\
{\left[\psi_{\text {out }}\right] } & =-\int_{\Omega_{\text {out }}} N_{m}^{T}[h]^{T}\left[\beta^{S}\right]^{-1} N_{\text {out }} d \Omega .
\end{aligned}
$$

3) Electrical Energy: By substituting the expressions of the electrical displacement from the piezoelectric constitutive equations (8) and (9) in (5) and rearranging the terms, the electrical energy of the PT is the sum of both contributions of the primary and secondary sides given by

$$
\begin{aligned}
W_{e, \text { in }} & =\frac{1}{2}\left\{v_{\text {in }}\right\}^{T}\left[C_{\text {in }}\right]\left\{v_{\text {in }}\right\}+\frac{1}{2}\left\{v_{\text {in }}\right\}^{T}\left[\psi_{\text {in }}\right]\{\eta\} \\
W_{e, \text { out }} & =\frac{1}{2}\left\{v_{\text {out }}\right\}^{T}\left[C_{\text {out }}\right]\left\{v_{\text {out }}\right\}-\frac{1}{2}\left\{v_{\text {out }}\right\}^{T}\left[\psi_{\text {out }}\right]\{\eta\},
\end{aligned}
$$

where $\left[C_{\text {in }}\right]$ and $\left[C_{\text {out }}\right]$ are the primary and secondary piezoelectric "blocked" capacitance matrices respectively, defined by

$$
\begin{aligned}
{\left[C_{\text {in }}\right] } & =\int_{\Omega_{\text {in }}} N_{\text {in }}^{T}\left[\varepsilon^{S}\right] N_{\text {in }} d \Omega \\
{\left[C_{\text {out }}\right] } & =\int_{\Omega_{\text {out }}} N_{\text {out }}^{T}\left[\beta^{S}\right]^{-1} N_{\text {out }} d \Omega .
\end{aligned}
$$

4) Lagrangian: The expression of the Lagrange function of the piezoelectric transformer is obtained by substituting $T, U$, and $W_{e}$ in (2). Observing that the terms $\{\eta\}^{T}\left[\psi_{\text {in }}\right]$ 
$\left\{v_{\text {in }}\right\}$ and $\{\eta\}^{T}\left[\psi_{\text {out }}\right]\left\{v_{\text {out }}\right\}$ are scalar, the Lagrangian is reduced to the expression given by

$$
\begin{aligned}
L= & \frac{1}{2}\{\dot{\eta}\}^{T}[M]\{\dot{\eta}\}-\frac{1}{2}\{\eta\}^{T}[K]\{\eta\} \\
& +\{\eta\}^{T}\left[\psi_{\text {in }}\right]\left\{v_{\text {in }}\right\}-\{\eta\}^{T}\left[\psi_{\text {out }}\right]\left\{v_{\text {out }}\right\} \\
& +\frac{1}{2}\left\{v_{\text {in }}\right\}^{T}\left[C_{\text {in }}\right]\left\{v_{\text {in }}\right\}+\frac{1}{2}\left\{v_{\text {out }}\right\}^{T}\left[C_{\text {out }}\right]\left\{v_{\text {out }}\right\}
\end{aligned}
$$

where $[M]$ and $[K]$ are, respectively, the total modal mass and stiffness of the piezoelectric transformer given by

$$
\begin{aligned}
& {[M]=\left[M_{\text {in }}\right]+\left[M_{\text {out }}\right]=\int_{\Omega} \lambda_{m}^{T} \rho \lambda_{m} d \Omega} \\
& {[K]=\left[K_{\text {in }}\right]+\left[K_{\text {out }}\right] .}
\end{aligned}
$$

The equations of motion can now be derived by making the substitution for the Lagrangian (23) and the variational work terms (7) in (1). By integrating the kinetic energy by parts in time and rearranging the terms, the dynamic equilibrium of a PT defined by the independent quantities $\{\delta \eta\},\left\{\delta v_{\text {in }}\right\}$, and $\left\{\delta v_{\text {out }}\right\}$ can be obtained. By allowing arbitrary variations for $\{\eta\},\left\{v_{\text {in }}\right\}$, and $\left\{v_{\text {out }}\right\} 3$ matrix equations of motion are obtained:

$$
\left\{\begin{array}{l}
{[M]\{\ddot{\eta}\}+[K]\{\eta\}=\left[\psi_{\text {in }}\right]\left\{v_{\text {in }}\right\}-\left[\psi_{\text {out }}\right]\left\{v_{\text {out }}\right\}} \\
{\left[\psi_{\text {in }}\right]^{T}\{\eta\}+\left[C_{\text {in }}\right]\left\{v_{\text {in }}\right\}=\left\{q_{\text {in }}\right\}} \\
{\left[\psi_{\text {out }}\right]^{T}\{\eta\}=\left[C_{\text {out }}\right]\left\{v_{\text {out }}\right\}+\left\{q_{\text {out }}\right\} .}
\end{array}\right.
$$

The dynamic solution is based on the generalized coordinates, which are the modal amplitude and the applied and received voltages. In the next part, the study of the free vibrations of a piezoelectric transformer is undertaken.

\section{B. Free Vibrations of Piezoelectric Transformers}

Mechanically, a piezoelectric medium is close to a "classic" elastic medium. Because of that, the methods of mechanical studies of linear elastic problems [7] can be adapted to linear piezoelectric problems. The eigenvalue problem of the dynamic equilibrium of a PT free of all external influences is considered. The assumption of a harmonic time evolution of the mechanical and electrical quantities can be made because the piezoelectric material is supposed to be linear. The displacement field and the electrical potential can be expressed with the following form:

$$
\begin{aligned}
u_{i}\left(x_{j}, t\right) & =u_{i}\left(x_{j}\right) \cos (\omega t) \\
\phi\left(x_{j}, t\right) & =\phi\left(x_{j}\right) \cos (\omega t) \quad \text { for } i, j=1,2,3 .
\end{aligned}
$$

The elastic and electrical behaviors of PT can be explained by the linear theory of the piezoelectricity. According to [8], a piezoelectric medium is governed by the local equations given by the Newton's and Gauss's electrostatic laws:

$$
\left\{\begin{array}{l}
\operatorname{div}\{T\}=\rho\{\ddot{u}\} \\
\operatorname{div}\{D\}=0 \text { in } \Omega .
\end{array}\right.
$$

To these equations showing the dynamic equilibrium of the linear piezoelectric continuum the boundary conditions must be added. Mechanically, assuming that the acoustic impedance of air is neglected, a traction-free condition is considered at the surface of the PT:

$$
T_{i j} n_{i}=0 \text { on } \Sigma,
$$

where $n_{i}$ denotes the components of the unit normal to the surface. Electrically, if the appropriate dielectric constant of the material is large compared with the dielectric constant of air, the boundary condition becomes approximately:

$$
D_{i} n_{i}=0 \text { on } \Sigma .
$$

Thus, the homogeneous system of equations and the harmonic time evolution assumption define an eigenvalue problem. Its eigenvalues are in infinite number and can be represented by the following ordered pairs:

$$
\left\{\left\{v^{(n)}\right\}=\left[\begin{array}{c}
\left\{u^{(n)}\right\} \\
\phi^{(n)}
\end{array}\right], \omega_{(n)}\right\} .
$$

According to (27), (28), and (29), they verify individually the following equations:

$$
\left\{\begin{array}{ll}
\operatorname{div}\left\{T^{(n)}\right\}+\rho \omega_{(n)}^{2}\left\{u^{(n)}\right\} & =0 \\
\operatorname{div}\left\{D^{(n)}\right\} & =0 \\
T_{i j}^{(n)} n_{i} & \text { in } \Omega \\
D_{i}^{(n)} n_{i} & =0
\end{array} \text { on } \Sigma=1, \ldots, \infty .\right.
$$

This problem includes 4 scalar equations with the associated boundary conditions which depend on the modal pulsation $\omega_{(n)}$. It will consequently be impossible to determine the eigensolutions in a unique way: it corresponds to the indetermination on the modal amplitudes $\left\{v^{(n)}\right\}$.

According to [7], the eigenmodes associated with a multiple eigenfrequency are linearly independent and they can be consequently chosen to be orthogonal. To prove the orthogonality properties of the eigensolutions, the equilibrium equations in the volume of the $\mathrm{PT}$ verified by the eigenvector $\left\{v^{(n)}\right\}$ is multiplied by the transpose of the eigenvector $\left\{v^{(m)}\right\}$ and integrated over the volume:

$$
\int_{\Omega}\left\{v^{(m)}\right\}^{T}\left[\begin{array}{c}
\operatorname{div}\left\{T^{(n)}\right\}+\rho \omega_{(n)}^{2}\left\{u^{(n)}\right\} \\
\operatorname{div}\left\{D^{(n)}\right\}
\end{array}\right] d \Omega=0 .
$$

The equilibrium equation of eigenvector $\left\{v^{(m)}\right\}$ is treated in the same way by multiplying it by the transpose of the eigenvector $\left\{v^{(n)}\right\}$. By using the constitutive equations 
of the driving and receiving sections and the boundary conditions verified by the eigenvectors $\left\{v^{(n)}\right\}$ and $\left\{v^{(m)}\right\}$, respectively, 2 relations are obtained. By manipulating them, the normalization of the modal mass is achieved by the following relation:

$$
\int_{\Omega} \rho\left\{u^{(m)}\right\}^{T}\left\{u^{(n)}\right\} d \Omega=\delta_{n m}
$$

where $\delta_{n m}$ is the Kronecker's symbol. A second relation of orthogonality can also be deduced, given by (34), between the strain field and the electrical field in the piezoelectric transformer induced by 2 different eigenmodes.

$$
\begin{aligned}
\int_{\Omega_{\text {in }}}[ & \left.\left\{S^{(m)}\right\}^{T}\left[c^{E}\right]\left\{S^{(n)}\right\}+\left\{E^{(n)}\right\}^{T}\left[\varepsilon^{S}\right]\left\{E^{(m)}\right\}\right] d \Omega \\
& +\int_{\Omega_{\text {out }}}\left[\left\{S^{(m)}\right\}^{T}\left(\left[c^{D}\right]-[h]^{T}\left[\beta^{S}\right]^{-1}[h]\right)\left\{S^{(n)}\right\}\right. \\
& \left.+\left\{E^{(n)}\right\}^{T}\left[\beta^{S}\right]^{-1}\left\{E^{(m)}\right\}\right] d \Omega=\delta_{n m} \omega_{(n)}^{2}
\end{aligned}
$$

The relationships (33) and (34) are a priori valid for any geometry of piezoelectric transformer.

\section{Application to Rosen Piezoelectric TRANSFORMER}

To illustrate the previously established electromechanical method, a typical Rosen PT is chosen. In concrete terms, this type of PT consists of a single rectangular piece of piezoelectric ceramic material; the primary part is poled in the thickness direction, whereas the secondary part is poled in the length direction.

This architecture is driven by an ac voltage power supply applied to the driving part at a frequency close to the length extensional modes frequency. As a consequence, by the converse piezoelectric effect, a promoted longitudinal vibration is transmitted to the receiving part by mechanical coupling. An electrical potential is therefore induced by the direct piezoelectric effect at the same frequency [9]. This architecture is particularly dedicated to high-voltage step-up ratio and low-power applications. For that reason, the primary side is often constituted with multilayers, which allows an increase of voltage step-up ratio.

\section{A. Modeling of Rosen PT}

Fig. 1 is a schematic of a multilayer Rosen PT of length $L_{0}$, width $w$, and thickness $t$. The transformer is constituted by a driving part transversally poled and a longitudinally poled receiving part of length $L_{1}$ and $L_{2}$, respectively. The origin of the coordinate system is chosen at the center of the interface between the primary and secondary portions. The driving section $-L_{1}<x_{1}<0$ is made of $m$ layers with $e / m$ thickness. In the receiving section $0<x_{1}$ $<L_{2}$, the output electrode at the end $x_{1}=L_{2}$ is connected at the load resistance $R_{L}$.

1) Definition of Lagrangian: To access a simple analytical solution and a representative description of electro-

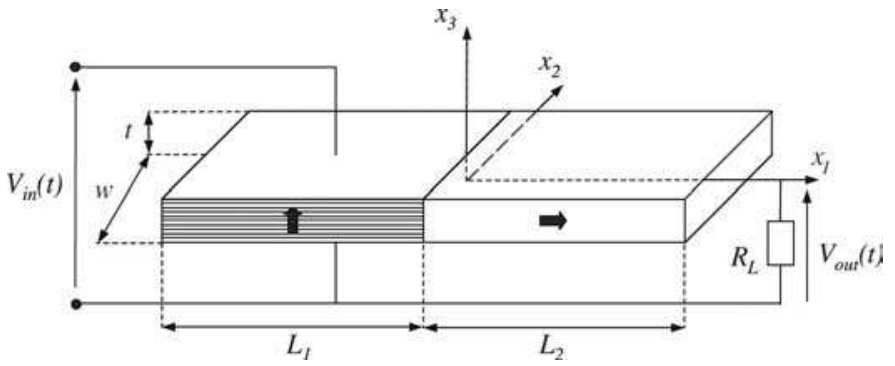

Fig. 1. Structure of classical Rosen transformer.

dynamical behavior of the PT, the multimodal model is derived from the following assumptions:

1) Because the optimum performance of this structure is achieved for longitudinal modes, the vibratory analysis will only deal with extensional modes, so the Rosen PT is considered as a thin sheet in extension motion.

2) The Rosen-type PT is considered as a thin ceramic rod with rectangular cross section in extensional motion in the axial direction $x_{1}$ [8]. The length $L_{0}$ is assumed to be much longer than the width $w$, and the thickness $t$ is much thinner than the width to satisfy thin plate approximation: $L_{0} \gg w \gg t$. Moreover, the electrodes' thickness is also neglected.

3) The state of 1-D stress is considered.

4) The boundary conditions for the Rosen PT are traction-free.

5) In first approach, the nonhomogeneous property along $x_{1}$ is considered, i.e., the difference of stiffness between the primary and secondary parts is taken into account.

Because the axes of poling are different in the primary and secondary sides, the material matrices for each ceramic have to be written by respecting the local coordinate system. In this case, for the ceramic poled in the $x_{3}$ direction, the material matrices of the driving portion take the following expressions [10]:

$$
\begin{gathered}
\left(\begin{array}{ccccccc}
c_{11}^{E} & c_{12}^{E} & c_{13}^{E} & 0 & 0 & 0 \\
c_{12}^{E} & c_{11}^{E} & c_{13}^{E} & 0 & 0 & 0 \\
c_{13}^{E} & c_{13}^{E} & c_{33}^{E} & 0 & 0 & 0 \\
0 & 0 & 0 & c_{44}^{E} & 0 & 0 \\
0 & 0 & 0 & 0 & c_{44}^{E} & 0 \\
0 & 0 & 0 & 0 & 0 & c_{66}^{E}
\end{array}\right) \\
\left(\begin{array}{cccccc}
0 & 0 & 0 & 0 & e_{15} & 0 \\
0 & 0 & 0 & e_{15} & 0 & 0 \\
e_{31} & e_{31} & e_{33} & 0 & 0 & 0
\end{array}\right),\left(\begin{array}{ccc}
\varepsilon_{11}^{S} & 0 & 0 \\
0 & \varepsilon_{11}^{S} & 0 \\
0 & 0 & \varepsilon_{33}^{S}
\end{array}\right) .
\end{gathered}
$$

For the ceramic poled in the $x_{1}$ direction, which is used to define the receiving section, the material matrices can be obtained from the previous matrices by rotating rows and columns properly. They are expressed as 


$$
\begin{gathered}
\left(\begin{array}{cccccc}
c_{33}^{D} & c_{13}^{D} & c_{13}^{D} & 0 & 0 & 0 \\
c_{13} & c_{11}^{D} & c_{12}^{D} & 0 & 0 & 0 \\
c_{13}^{D} & c_{12}^{D} & c_{11}^{D} & 0 & 0 & 0 \\
0 & 0 & 0 & c_{66}^{D} & 0 & 0 \\
0 & 0 & 0 & 0 & c_{44}^{D} & 0 \\
0 & 0 & 0 & 0 & 0 & c_{44}^{D}
\end{array}\right) \\
\left(\begin{array}{ccccccc}
h_{33} & h_{31} & h_{31} & 0 & 0 & 0 \\
0 & 0 & 0 & 0 & 0 & h_{15} \\
0 & 0 & 0 & 0 & h_{15} & 0
\end{array}\right),\left(\begin{array}{ccc}
\beta_{33}^{S} & 0 & 0 \\
0 & \beta_{11}^{S} & 0 \\
0 & 0 & \beta_{11}^{S}
\end{array}\right) .
\end{gathered}
$$

From these assumptions and geometrical considerations, the constitutive relations of the primary and secondary portions can be simplified. Indeed, after assumption 3 is made, the PT is assumed to undergo an uniaxial stress along the axis $x_{1}$ and to be free of shear stresses. Thus, the stress and strain vectors are reduced to one component:

$$
\begin{aligned}
& \{T\}=\left[\begin{array}{llllll}
T_{1} & 0 & 0 & 0 & 0 & 0
\end{array}\right]^{T} \\
& \{S\}=\left[\begin{array}{llllll}
S_{1} & 0 & 0 & 0 & 0 & 0
\end{array}\right]^{T} .
\end{aligned}
$$

Moreover, as each layer is assumed to be supplied by a sinusoidal voltage, the electrical field is oriented parallel to the axis (3), in the same direction of the depolarizing field. Both electrical field and electrical displacement are consequently reduced to one component:

$$
\begin{aligned}
& \{D\}=\left[\begin{array}{lll}
0 & 0 & D_{3}
\end{array}\right]^{T} \\
& \{E\}=\left[\begin{array}{lll}
0 & 0 & E_{3}
\end{array}\right]^{T} .
\end{aligned}
$$

This leads to the following reduced constitutive relationships of the primary part given by

$$
\begin{aligned}
T_{1} & =\tilde{c}_{11} S_{1}-\tilde{e}_{31} E_{3} \\
D_{3} & =\tilde{e}_{31} S_{1}+\tilde{\varepsilon}_{33} E_{3} .
\end{aligned}
$$

The tilde symbol on the coefficients of the piezoelectric material is a note to use the specific values of the transverse coupling mode. These coefficients are given in Table $\mathrm{I}$, where $k_{31}$ is the material coupling factor relative to the transverse mode.

Furthermore, assuming that the electrical field is constant as well as the thickness, the operator matrix $L_{\text {in }}$ can be simply defined by

$$
L_{\text {in }}=\left[-\frac{m}{t}\right]
$$

and as each layer is supplied by the applied voltage $V_{\text {in }}(t)$, the primary electrical field is defined as

$$
\{E\}=N_{\text {in }} V_{\text {in }}(t) \text { with } N_{\text {in }}=\left[\begin{array}{lll}
0 & 0 & -m / t
\end{array}\right]^{T} .
$$

Concerning the receiving section, the insulating and without free charges ceramic assures that the components of
TABle I. Piezoelectric Material Coefficients of the Transverse and Longitudinal Coupling Modes.

$$
\begin{aligned}
& \tilde{c}_{11}=\frac{1}{s_{11}^{E}} \\
& \tilde{c}_{33}=\frac{1}{s_{33}^{E}\left(1-k_{33}^{2}\right)} \\
& \tilde{e}_{31}=\frac{d_{31}}{s_{11}^{E}} \\
& \tilde{h}_{33}=\frac{d_{33}}{s_{33}^{E} \epsilon_{33}^{T}\left(1-k_{33}^{2}\right)} \\
& \tilde{\epsilon}_{33}=\epsilon_{33}^{T}\left(1-k_{31}^{2}\right) \\
& \tilde{\beta}_{33}=\frac{1}{\epsilon_{33}^{T}\left(1-k_{33}^{2}\right)}
\end{aligned}
$$

electrical displacement along the axes (2) and (3) are equal to zero and, according to Gauss's law, the component $D_{1}$ is constant. Both electrical field and electrical displacement are consequently reduced to one element:

$$
\begin{aligned}
& \{D\}=\left[\begin{array}{lll}
D_{1} & 0 & 0
\end{array}\right]^{T} \\
& \{E\}=\left[\begin{array}{lll}
E_{1} & 0 & 0
\end{array}\right]^{T} .
\end{aligned}
$$

This leads to the following reduced constitutive relationships of the secondary part:

$$
\begin{aligned}
T_{1} & =\tilde{c}_{33} S_{1}-\tilde{h}_{33} D_{1} \\
E_{1} & =-\tilde{h}_{33} S_{1}+\tilde{\beta}_{33} D_{1} .
\end{aligned}
$$

The tilde symbol on the coefficients of the piezoelectric material is a note to use the specific values of the longitudinal coupling mode. These coefficients are given in Table I, where $k_{33}$ is the material coupling factor relative to the longitudinal mode.

In a similar way, the operator matrix $L_{\text {out }}$ can be simply defined

$$
L_{\text {out }}=\left[-\frac{1}{L_{2}}\right]
$$

and as the piezoelectric transformer produces an output voltage $V_{\text {out }}(t)$, the secondary electrical field is defined as follows:

$$
\{E\}=N_{\text {out }} V_{\text {out }}(t) \text { with } N_{\text {out }}=\left[\begin{array}{lll}
-1 / L_{2} & 0 & 0
\end{array}\right]^{T} .
$$

2) Vibratory Analysis: To apply the previously detailed electromechanical modeling to extract a multimodal model, a preliminary vibratory analysis is necessary to characterize the displacement field of extensional modes.

The vibratory study follows the 1-D model of a bar in extension explained in [10]. Furthermore, the number of considered longitudinal modes will be limited to minimize the influence of transverse modes (assumption 2). Accord- 
ing to assumption 5, the Rosen PT is the association of a primary and secondary parts considered as continuous beams of length $L_{1}$ (resp. $L_{2}$ ), density $\rho$, and stiffness $\tilde{c}_{11}$ (resp. $\left.\tilde{c}_{33}\right)$. From (27) and the reduced constitutive equations (39) and (43), the following dynamic equilibrium equation in the assumption of linear piezoelectricity is obtained:

$$
\begin{aligned}
& \frac{\partial^{2} u_{1}}{\partial x_{1}^{2}}=\frac{\rho}{\tilde{c}_{11}} \frac{\partial^{2} u_{1}}{\partial t^{2}} \text { for }-L_{1}<x_{1}<0 \\
& \frac{\partial^{2} u_{1}}{\partial x_{1}^{2}}=\frac{\rho}{\tilde{c}_{33}} \frac{\partial^{2} u_{1}}{\partial t^{2}} \text { for } 0<x_{1}<L_{2} .
\end{aligned}
$$

According to the free vibrations assumption (assumption 4 ), the driving and receiving electrodes are considered as short and open, respectively. This means that the driving voltage $V_{\text {in }}(t)$ is equal to zero. Hence, from (43), the expression of the electrical potential along the structure can be obtained:

$$
\phi^{(n)}\left(x_{1}, t\right)= \begin{cases}0, & -L_{1}<x_{1}<0 \\ \tilde{h}_{33} u_{1}\left(x_{1}, t\right)+A(t) x_{1}+B(t), & 0<x_{1}<L_{2}\end{cases}
$$

and $A(t)$ and $B(t)$ are 2 integration constants which may still be functions of time. Physically, $A(t)$ represents the output electric charge and as a consequence the current on the receiving electrode at $x_{1}=L_{2}[10]$. Thus, as the latter is supposed to be open for free vibrations study, $A(t)$ is considered to be equal to zero for vibratory analysis.

Concerning the interface conditions, the displacement, the stress, and the electrical potential verify the following continuity relations at the junction of both sections, $x_{1}=$ 0 :

$$
\begin{aligned}
u_{1}\left(x_{1}\right. & \left.=0^{-}, t\right)=u_{1}\left(x_{1}=0^{+}, t\right) \\
T_{1}\left(x_{1}\right. & \left.=0^{-}, t\right)=T_{1}\left(x_{1}=0^{+}, t\right) \\
\phi\left(x_{1}\right. & \left.=0^{-}, t\right)=\phi\left(x_{1}=0^{+}, t\right) .
\end{aligned}
$$

Furthermore the free-free ends boundary conditions (assumption 4) can be written as follows:

$$
\begin{gathered}
T_{1}\left(x_{1}=-L_{1}, t\right)=\tilde{c}_{11} \frac{\partial u_{1}}{\partial x_{1}}\left(x_{1}=-L_{1}, t\right)=0 \\
T_{1}\left(x_{1}=L_{2}, t\right)=\tilde{c}_{33} \frac{\partial u_{1}}{\partial x_{1}}\left(x_{1}=L_{2}, t\right)=0 .
\end{gathered}
$$

To analyze the free vibrations, the harmonic motion assumption $u_{1}\left(x_{1}, t\right)=U^{(n)}\left(x_{1}\right) \cos (\omega t)$ is considered. Thus, (46) is modified into

$$
\begin{aligned}
& \frac{d^{2} U^{(n)}}{d x_{1}^{2}}+k_{1}^{(n)} U^{(n)}=0 \text { for }-L_{1}<x_{1}<0 \\
& \frac{d^{2} U^{(n)}}{d x_{1}^{2}}+k_{2}^{(n)} U^{(n)}=0 \text { for } 0<x_{1}<L_{2},
\end{aligned}
$$

which admits the general solution $(i=1,2)$

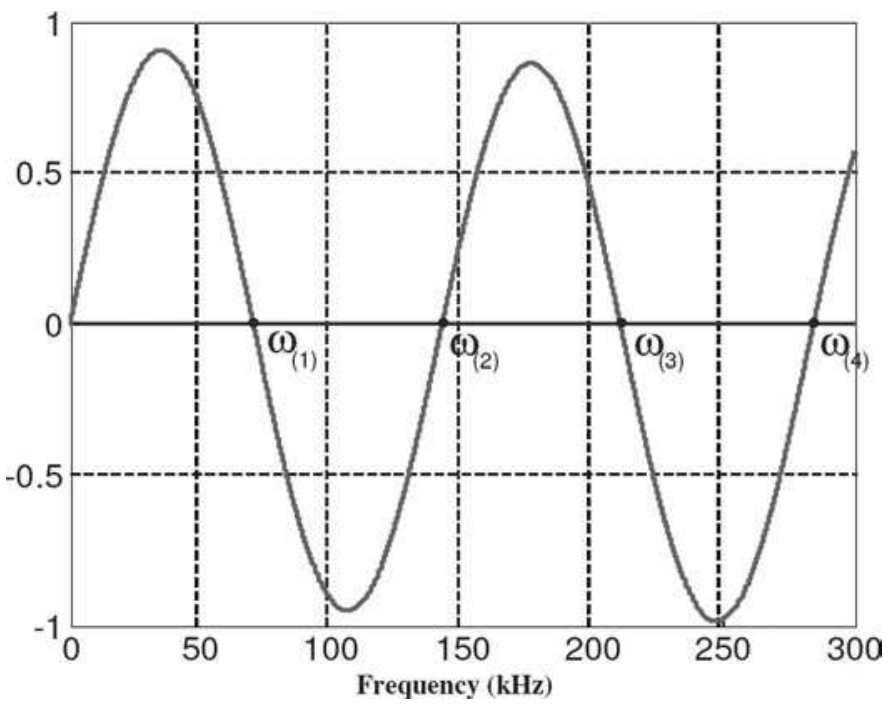

Fig. 2. Determination of eigenfrequencies by graphic resolution.

$$
U^{(n)}\left(x_{1}\right)=A_{i} \cos \left(k_{i}^{(n)} x_{1}\right)+B_{i} \sin \left(k_{i}^{(n)} x_{1}\right),
$$

where $k_{1}^{(n)}$ and $k_{2}^{(n)}$ are the wave vectors of the $n$th mode in the primary and secondary parts, respectively, with the following expressions

$$
\begin{aligned}
& k_{1}^{(n)}=\omega_{(n)} \sqrt{\frac{\rho}{\tilde{c}_{11}}}=\omega_{(n)} \sqrt{\rho s_{11}^{E}} \\
& k_{2}^{(n)}=\omega_{(n)} \sqrt{\frac{\rho}{\tilde{c}_{33}}}=\omega_{(n)} \sqrt{\rho s_{33}^{E}\left(1-k_{33}^{2}\right)},
\end{aligned}
$$

and finally $\omega_{(n)}$ is the $n$th root of the frequency equation given by (A1) and obtained by the determination of constants $A_{i}$ and $B_{i}$. A graphic solution of the frequency equation is presented in Fig. 2. The general solution of the proposed eigenvalue problem consequently takes the form of (A2). Therefore, in the interests of an appropriate choice of generalized coordinate, the eigenfunction $U^{(n)}$ must be normalized to match the mechanical generalized coordinate with the maximum amplitude of the displacement. The selected criterion of normalization affects the modal mass according to (33). The constant $U_{0}^{(n)}$ consequently takes the form given by (A3) where the symbol sinc represents the unnormalized sinc function. Note that $U_{0}^{(n)}$ is inversely proportional to the square root of a mass and in this instance, if the multiplying constant $\sqrt{M_{0}}$, where $M_{0}$ is given by (53), is added to normalize the eigenfunction $U^{(n)}$, the mechanical generalized coordinate will be the maximum amplitude of the displacement.

$$
M_{0}=\frac{1}{2} \rho w t L
$$

Thus, for applied frequencies close to mechanical resonant frequency, the displacement field of the Rosen-type piezoelectric transformer can be finally defined by:

$$
u_{1}\left(x_{1}, t\right)=\hat{U}^{(n)}\left(x_{1}\right) \eta_{\omega}^{(n)}(t)
$$




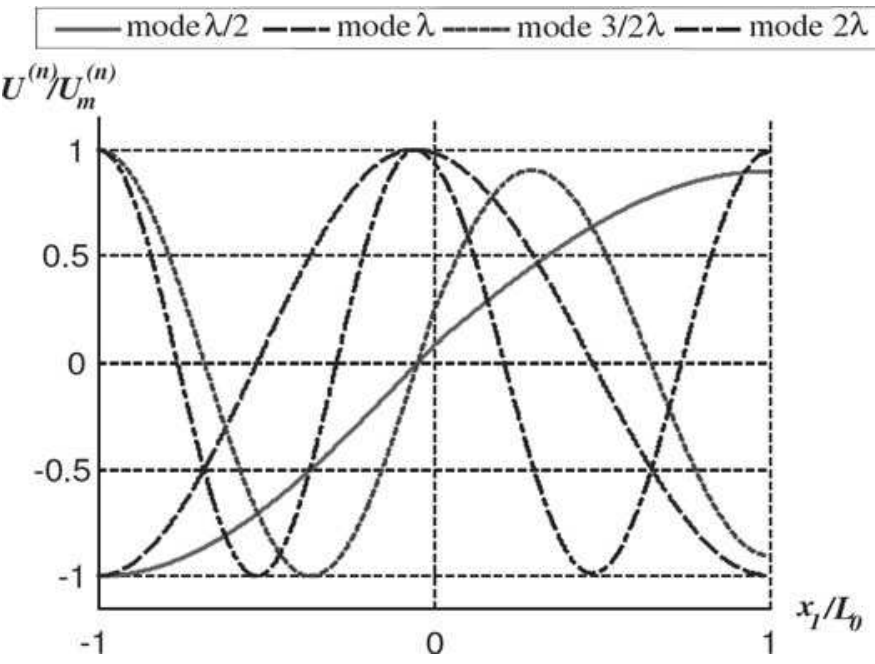

Fig. 3. Normalized mechanical shape of the first 4 extensional modes.

where $\eta_{\omega}^{(n)}$ and $\hat{U}^{(n)}$ are, respectively, the mechanical generalized coordinates representing the maximum amplitude of the displacement and the normalized eigenfunction of the $n$th extensional mode with:

$$
\hat{U}^{(n)}\left(x_{1}\right)=\sqrt{M_{0}} U^{(n)}\left(x_{1}\right) .
$$

As the consequence, the assumed mechanical mode shape matrix $\lambda_{m}$ can be defined as

$$
\lambda_{m}=\left(\begin{array}{ccc}
\hat{U}^{(1)}\left(x_{1}\right) & \cdots & \hat{U}^{(n)}\left(x_{1}\right) \\
0 & \cdots & 0 \\
0 & \cdots & 0
\end{array}\right) .
$$

The normalized mechanical displacement of the first 4 longitudinal modes for a Rosen PT with equal primary and secondary lengths are shown in Fig. $3 ; \lambda$ represents the wavelength of the vibratory phenomenon. Note that the location of the nodal point is sensitive to the difference of stiffness between the driving and receiving parts. In fact, in the case that compliances of both sections verify the inequality $s_{11}^{E}<s \frac{E}{33}\left(1-k_{33}^{2}\right)$, the nodal point of the first extensional mode appears in the left half where the material is more rigid in the $x_{1}$ direction. In Fig. 4 , the normalized electrical potential of the first 4 extensional modes functions of $x_{1}$ are represented. $\phi^{(1)}$ and $\phi^{(2)}$ rise in receiving part. That is why Rosen-type PT are generally designed to operate at the first 2 extensional modes [9].

3) Application of Hamilton's Principle: After the vibratory analysis is made, the Hamilton's principle is applied. According to the general analytical formulation, the Lagrangian of the Rosen PT can be expressed by (23). By calculating the integral in (24) and using the criterion of normalization (33), the modal mass matrix of the Rosen $\mathrm{PT}$ is diagonal and reads

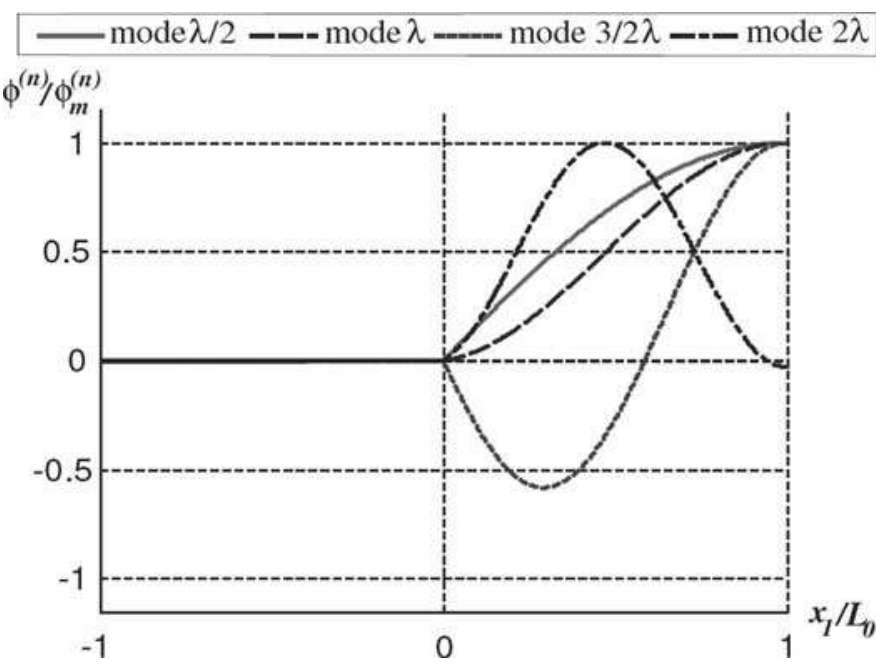

Fig. 4. Normalized electrical potential of the first 4 extensional modes.

$$
[M]=\left(\begin{array}{cccc}
M^{(1)} & 0 & \cdots & 0 \\
0 & \ddots & \ddots & \vdots \\
\vdots & \ddots & \ddots & 0 \\
0 & \cdots & 0 & M^{(n)}
\end{array}\right)
$$

where the modal mass of the $i$ th mode is equal to $M_{0}$. The total stiffness matrix can be calculated from (19) and (24). By using the equation of normalization (34), the diagonalization of the stiffness matrix is achieved as follows:

$$
[K]=\left(\begin{array}{cccc}
K^{(1)} & 0 & \cdots & 0 \\
0 & \ddots & \ddots & \vdots \\
\vdots & \ddots & \ddots & 0 \\
0 & \cdots & 0 & K^{(n)}
\end{array}\right)
$$

where the modal stiffness of the $i$ th mode is given by (59), where the piezoelectric material coefficient of the transverse and longitudinal coupling modes (Table I) have been used.

$$
\begin{aligned}
K^{(i)}= & \left(\hat{U}_{0}^{(i)}\right)^{2}\left[\frac{w t}{2 L_{1} s_{11}^{E}}\left(k_{1}^{(i)} L_{1}\right)^{2} \frac{1-\operatorname{sinc}\left(2 k_{1}^{(i)} L_{1}\right)}{\cos ^{2}\left(k_{1}^{(i)} L_{1}\right)}\right. \\
& \left.+\frac{w t}{2 L_{2} s_{33}^{E}\left(1-k_{33}^{2}\right)}\left(k_{2}^{(i)} L_{2}\right)^{2} \frac{1-\operatorname{sinc}\left(2 k_{2}^{(i)} L_{2}\right)}{\cos ^{2}\left(k_{2}^{(i)} L_{2}\right)}\right]^{(59)}
\end{aligned}
$$

The electromechanical coupling matrices of the driving and receiving sections take the form of a column vector as follows:

$$
\left[\psi_{\text {in }}\right]=\left(\begin{array}{c}
\psi_{\text {in }}^{(1)} \\
\vdots \\
\psi_{\text {in }}^{(n)}
\end{array}\right), \quad\left[\psi_{\text {out }}\right]=\left(\begin{array}{c}
\psi_{\text {out }}^{(1)} \\
\vdots \\
\psi_{\text {out }}^{(n)}
\end{array}\right),
$$

where the modal electromechanical factor of the $i$ th mode of the primary and secondary portions are given by 


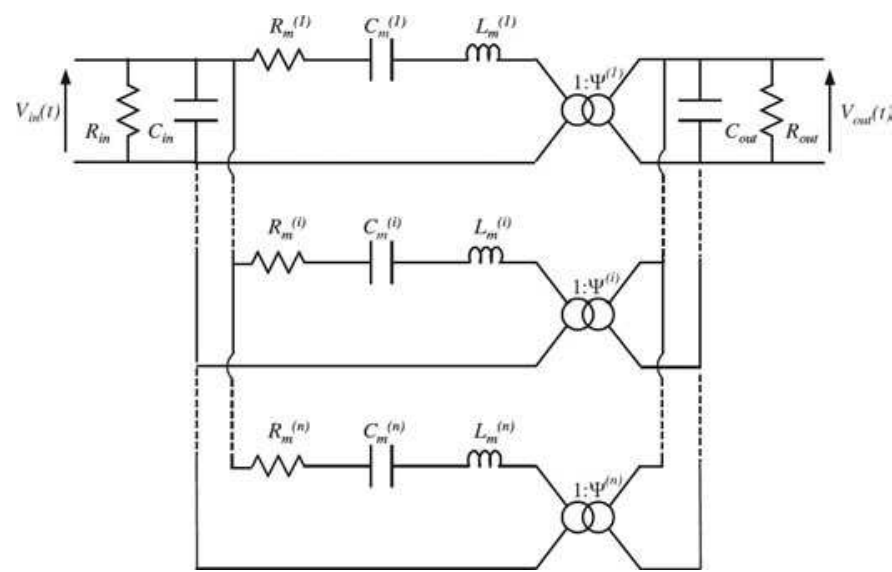

Fig. 5. Equivalent electrical circuit of Rosen piezoelectric transformer.

$$
\begin{aligned}
{\left[\psi_{\text {in }}^{(i)}\right] } & =\hat{U}_{0}^{(i)} m w \frac{d_{31}}{s_{11}^{E}} \frac{1-\cos \left(k_{1}^{(i)} L_{1}\right)}{\cos \left(k_{1}^{(i)} L_{1}\right)} \\
{\left[\psi_{\text {out }}^{(i)}\right] } & =\hat{U}_{0}^{(i)} \frac{w t}{L_{2}} \frac{d_{33}}{s_{33}^{E}} \frac{1-\cos \left(k_{2}^{(i)} L_{2}\right)}{\cos \left(k_{2}^{(i)} L_{2}\right)} .
\end{aligned}
$$

The input and output "blocked" capacitances of the Rosen PT can be simply defined by a scalar as

$$
\begin{aligned}
{\left[C_{\text {in }}\right] } & =C_{\text {in }}=m^{2} \frac{w L_{1} \varepsilon_{33}^{T}\left(1-k_{31}^{2}\right)}{e} \\
{\left[C_{\text {out }}\right] } & =C_{\text {out }}=\frac{w t \varepsilon_{33}^{T}\left(1-k_{33}^{2}\right)}{L_{2}} .
\end{aligned}
$$

By applying Hamilton's principle, the dynamic equilibrium of a Rosen $\mathrm{PT}$ is governed by one matrix equation and 2 scalar relations:

$$
\left\{\begin{array}{l}
{[M]\{\ddot{\eta}\}+[K]\{\eta\}=\left[\psi_{\text {in }}\right] V_{\text {in }}-\left[\psi_{\text {out }}\right] V_{\text {out }}} \\
{\left[\psi_{\text {in }}\right]^{T}\{\eta\}+C_{\text {in }} V_{\text {in }}=q_{\text {in }}} \\
{\left[\psi_{\text {out }}\right]^{T}\{\eta\}=C_{\text {out }} V_{\text {out }}+q_{\text {out }} .}
\end{array}\right.
$$

The second-order mechanical equation shows a resonant phenomenon whose characteristic modal frequency $f_{r}^{(i)}$ takes the form

$$
f_{r}^{(i)}=\frac{1}{2 \pi} \sqrt{\frac{K^{(i)}}{M^{(i)}}} .
$$

By manipulating (63), the Rosen-type PT's vibratory behavior can be represented by an equivalent circuit (Fig. 5) where resistances have been added to take into account the dielectric and mechanical losses. The modal mechanical losses are represented by a resistance $R_{m}^{(i)}$ which is inversely proportional to the mechanical quality factor $Q_{m}$ of piezoelectric material.

The dielectric losses at the primary and secondary sections are respectively symbolized by the resistances $R_{\text {in }}$ and $R_{\text {out }}$, estimated by the dielectric loss angle. The mod- al transformer ratio of the Rosen PT is also introduced and is given by

$$
\psi^{(i)}=\frac{\psi_{\text {in }}^{(i)}}{\psi_{\text {out }}^{(i)}} .
$$

\section{B. Modeling of Simplified Homogeneous PT}

A current simplified analytical model of the electromechanical behavior of a Rosen-type PT is to consider the structure as a homogeneous medium. In this section, the presented method is newly applied from this additional assumption. As a consequence, the stiffness of the primary side close to the one of the secondary part is implicitly supposed. This assumption takes the following analytical form:

$$
\frac{\tilde{c}_{33}}{\tilde{c}_{11}}=\frac{s_{11}^{E}}{s_{33}^{E}\left(1-k_{33}^{2}\right)} \approx 1 .
$$

Thus, to determine the electrodynamical characteristics, the application of Hamilton's principle requires a new vibratory analysis to obtain the displacement field. Consequently, consider the Rosen PT as a continuous and homogeneous beam of length $L_{0}$, density $\rho$, and stiffness $c$. Newton's second law applied on a slice of length $d x_{1}$ gives, in the assumption of linear elasticity,

$$
\frac{\partial^{2} u_{1}}{\partial x_{1}^{2}}=\frac{\rho}{c} \frac{\partial^{2} u_{1}}{\partial t^{2}}
$$

Following the example of the previous vibratory analysis, by making the free-free ends boundary conditions and harmonic motion assumptions, the solution of free vibrations problem can be expressed as follows:

$$
V^{(n)}=\left[\begin{array}{l}
U_{0}^{(n)}\left[\cos \left(k^{(n)} x_{1}\right)-\tan \left(k^{(n)} L_{1}\right) \sin \left(k^{(n)} x_{1}\right)\right] \\
\phi_{0}^{(n)}\left[\cos \left(k^{(n)} x_{1}\right)-1-\tan \left(k^{(n)} L_{1}\right) \sin \left(k^{(n)} x_{1}\right)\right]
\end{array}\right],
$$

where $k^{(n)}$ is the wave vectors of the $n$th mode in the Rosen PT. Contrary to the previous study, the wave vector has an analytical expression which is obtained by calculating the constants of integration:

$$
k^{(n)}=\frac{n \pi}{L_{0}} .
$$

Therefore, to match the mechanical generalized coordinate to the maximum amplitude of the displacement, the normalization of the displacement field can be made just by substituting $k_{1}^{(n)}$ and $k_{2}^{(n)}$ by $k^{(n)}$ in the expression of the constant $U_{0}^{(n)}$. The same will be true of the calculus of the modal matrices (mass, stiffness, and electromechanical factor) during the application of Hamilton's principle. The normalized mechanical displacements of the first 4 longi- 


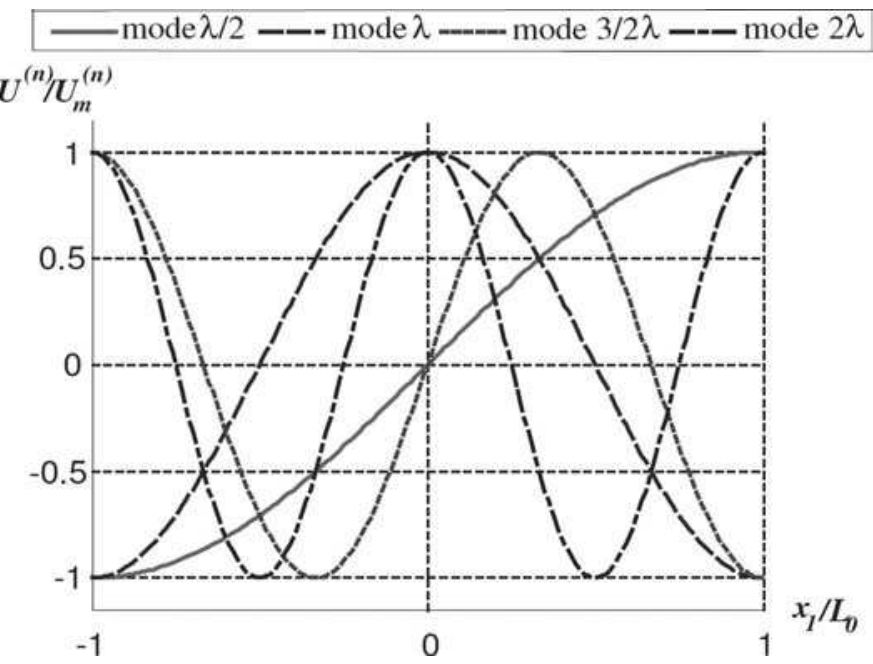

Fig. 6. Normalized mechanical shape of the first 4 modes.

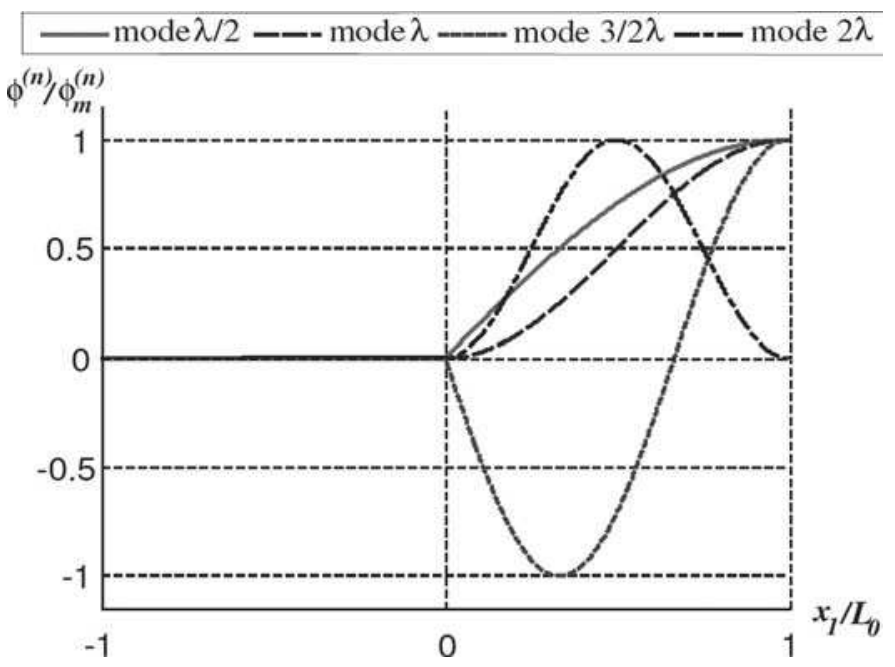

Fig. 7. Normalized electrical potential of the first 4 modes.

table iI. Noliac Ceramic Multilayer Transformer Rosen Piezoelectric Transformer's Geometric Parameters and Material Properties.

\begin{tabular}{llcc}
\hline & Definition & Value & Unity \\
\hline$L_{1}$ & Primary length & 12 & $\mathrm{~mm}$ \\
$L_{2}$ & Secondary length & 13 & $\mathrm{~mm}$ \\
$w$ & Width & 5 & $\mathrm{~mm}$ \\
$t$ & Thickness & 1.7 & $\mathrm{~mm}$ \\
$m$ & Primary layers & 16 & \\
$\rho$ & Mass density & 7600 & $\mathrm{~kg} / \mathrm{m}^{3}$ \\
$s_{11}^{E}$ & Transversal compliance at constant E & $1.256 e^{-11}$ & $\mathrm{~m}^{2} / \mathrm{N}$ \\
$s_{33}^{E}$ & Longitudinal compliance at constant E & $1.610 e^{-11}$ & $\mathrm{~m}^{2} / \mathrm{N}$ \\
$d_{31}$ & Transversal piezoelectric coefficient & $-1.329 e^{-10}$ & $\mathrm{~m} / \mathrm{N}$ \\
$d_{33}^{T}$ & Longitudinal piezoelectric coefficient & $3.086 e^{-10}$ & $\mathrm{~m} / \mathrm{N}$ \\
$\epsilon_{33}^{T}$ & $1454 \epsilon_{0}$ & $\mathrm{~F} / \mathrm{m}$ \\
$k_{31}$ & Permittivity at constant T & 0.330 & \\
$k_{33}$ & Transversal coupling factor & 0.678 & \\
$Q_{m}$ & Longitudinal coupling factor & 1000 & \\
$\tan \delta$ & Mechanical quality factor & 0.003 & \\
\hline
\end{tabular}

tudinal modes are shown in Fig. 6 for a Rosen PT with equal primary and secondary lengths. It can be noted that, contrary to the previous vibratory analysis, the assumed homogeneity along the $x_{1}$ direction makes normalized mechanical displacement of extensional modes centered in the middle of the structure. In Fig. 7, the normalized electrical potential of the first 4 modes functions of $x_{1}$ are represented.

Hereafter, comparison between the electrodynamical and electrical characteristics of both analytical models will be detailed.

\section{Comparisons of Both Models}

To check the relevance of both electromechanical models, a Noliac Ceramic Multilayer Transformer (CMT; Noliac Group, Kvistgaard, Denmark) is considered [11]. The piezoelectric material used in PT's processing is a lead zirconate titanate ceramic. The geometric parameters and the material properties are itemized in Table II.
According to Table II, modal electromechanical parameters of nonhomogeneous PT model can be calculated from (57)-(62). They are shown in Table III. For the homogeneous model, the modal characteristic parameters are obtained by simply substituting $k_{1}^{(n)}$ and $k_{2}^{(n)}$ by $k^{(n)}$ in (57)-(62). These parameters are detailed in Table IV.

In Tables III and IV, the series resonant frequencies at short and open receiving electrode are added. Their expressions take the form

$$
f_{s}=\frac{1}{2 \pi} \sqrt{\frac{K}{M}}, \quad f_{p}=\frac{1}{2 \pi} \sqrt{\frac{K}{M}\left(1+\frac{\psi_{\text {out }}^{2}}{K C_{\text {out }}}\right)} .
$$

Comparisons of results in Tables III and IV lead to several conclusions about accuracy and interest of these models. First, the vibratory behavior defined by the resonant frequencies, modal stiffness, and modal mass is quite similar in both cases. According to mass normalization assumption, both models modal mass is constant regardless of the vibratory mode. Second, significant differences 
TABle III. Modal Parameters of Nonhomogeneous Rosen-Type Piezoelectric Transformer

MODEL.

\begin{tabular}{lcccc}
\hline & Mode $\lambda / 2$ & Mode $\lambda$ & Mode $3 / 2 \lambda$ & Mode $2 \lambda$ \\
\hline$f_{s}[\mathrm{~Hz}]$ & 63686 & 123430 & 190790 & 247420 \\
$f_{p}[\mathrm{~Hz}]$ & 69276 & 139630 & 194520 & 247420 \\
$C_{\text {in }}[\mathrm{nF}]$ & 103.63 & 103.63 & 103.63 & 103.63 \\
$C_{\text {out }}[\mathrm{pF}]$ & 4.551 & 4.551 & 4.551 & 4.551 \\
$M[\mathrm{~g}]$ & 0.808 & 0.808 & 0.808 & 0.808 \\
$K[\mathrm{GN} / \mathrm{m}]$ & 0.129 & 0.486 & 1.161 & 1.952 \\
$L_{m}[\mathrm{mH}]$ & 0.823 & 0.285 & 1.569 & 287.9 \\
$C_{m}[\mathrm{nF}]$ & 7.584 & 5.833 & 0.443 & 0.001 \\
$\psi_{\text {in }}[\mathrm{C} / \mathrm{m}]$ & 0.990 & 1.683 & -0.717 & -0.053 \\
$\psi_{\text {out }}[\mathrm{C} / \mathrm{m}]$ & 0.0104 & -0.0249 & -0.0144 & 0.0006 \\
$\psi$ & 95.37 & -67.68 & 49.70 & -96.52 \\
\hline
\end{tabular}

TABle IV. Modal Parameters of Homogeneous Rosen-Type Piezoelectric Transformer Model.

\begin{tabular}{lcccc}
\hline & Mode $\lambda / 2$ & Mode $\lambda$ & Mode $3 / 2 \lambda$ & Mode $2 \lambda$ \\
\hline$f_{s}[\mathrm{~Hz}]$ & 60760 & 122120 & 182290 & 244220 \\
$f_{p}[\mathrm{~Hz}]$ & 70103 & 138600 & 184240 & 244230 \\
$C_{\text {in }}[\mathrm{nF}]$ & 103.63 & 103.63 & 103.63 & 103.63 \\
$C_{\text {out }}[\mathrm{pF}]$ & 4.551 & 4.551 & 4.551 & 4.551 \\
$M[\mathrm{~g}]$ & 0.808 & 0.808 & 0.808 & 0.808 \\
$K[\mathrm{GN} / \mathrm{m}]$ & 0.117 & 0.475 & 1.060 & 1.901 \\
$L_{m}[\mathrm{mH}]$ & 1.284 & 0.284 & 0.800 & 1143 \\
$C_{m}[\mathrm{nF}]$ & 5.343 & 5.976 & 0.953 & 0.0004 \\
$\psi_{\text {in }}[\mathrm{C} / \mathrm{m}]$ & -0.793 & 1.685 & 1.005 & -0.026 \\
$\psi_{\text {out }}[\mathrm{C} / \mathrm{m}]$ & -0.0133 & -0.0250 & 0.0101 & 0.0004 \\
$\psi$ & 59.54 & -67.52 & 98.66 & -67.52 \\
\hline
\end{tabular}

appear on electromechanical conversion factors except for the $(\lambda)$-mode. More generally, modal characteristics of both models are similar for the $(\lambda)$-mode. The weak influence of nonhomogeneous media on the even waveforms can give the proof of this noteworthy agreement.

Another significant difference is observed on the electrical potential repartition along the receiving part, particularly for the $(3 / 2 \lambda)$-mode. An obvious difference of the extremum values of the electrical potential appears on Figs. 4 and 7. These curves are obtained from equations (A1) and (68), respectively. This difference lies in the value of $k^{(n)}$ factor and $L_{1} / L_{2}$ ratio. The singular unit waveform on Fig. 7 appears in the case of consideration of homogeneous medium and equal primary and secondary lengths.

In intermediate conclusion about both models comparisons, it can be deduced that the choice of analytical approach and assumption considerations depend on the model's final objective. A simple model will be a sufficient approximation in the case of single mode consideration. The most complete model becomes necessary if a multimodal approach is required for optimization problems without arbitrary selected mode or in the case that the electrical potential repartition is a considered point of view.

In following section, previous analytical values will be compared with results obtained by finite elements method and experimental results.

\section{Numerical and Experimental Validation}

To verify the Rosen-type PT analytical electrodynamical model, a numerical study and an experimental identification were undertaken.

The experimental identification of the electrical equivalent circuit of the CMT Noliac transformer has been led for the first 4 longitudinal vibratory modes and previously undertaken in [12]. This identification is based on admittance measurements with Agilent HP 4294A impedance analyzer (Agilent Technologies Inc., Santa Clara, CA).

By two successive characterizations with the input and output terminal shorted, respectively, the electrical equivalent circuit is fully identified [13].

The following part is dedicated to briefly introducing the numerical method. The aim of this approach is to validate the theoretical modal mechanical shapes and electrical potentials and to extract the electromechanical parameters of the PT.

\section{A. Development of Numerical Method}

The method to characterize the double electromechanical conversion is based on a modal analysis and energy considerations using a finite element method with the ANSYS software. The modal study concerns the longitudinal mode of a PT composed of 2 rectangular piezoelectric ceramics of length $L_{1}$ (resp. $L_{2}$ ), width $w$, thickness $t$, and 
Table V. Modal Parameters of Numerical Rosen-Type Piezoelectric Transformer Model.

\begin{tabular}{lcccc}
\hline & Mode $\lambda / 2$ & Mode $\lambda$ & Mode $3 / 2 \lambda$ & Mode $2 \lambda$ \\
\hline$f_{s}[\mathrm{~Hz}]$ & 61883 & 124873 & 203569 & 265276 \\
$f_{p}[\mathrm{~Hz}]$ & 704468 & 141249 & 206495 & 265279 \\
$M[\mathrm{~g}]$ & 0.973 & 0.762 & 0.600 & 0.654 \\
$K[\mathrm{GN} / \mathrm{m}]$ & 0.191 & 0.600 & 1.008 & 1.818 \\
$L_{m}[\mathrm{mH}]$ & 0.852 & 0.333 & 2.936 & 131.8 \\
$C_{m}[\mathrm{nF}]$ & 5.984 & 3.814 & 0.202 & 0.003 \\
$\psi_{\text {in }}[\mathrm{C} / \mathrm{m}]$ & -1.068 & 1.513 & -0.451 & -0.070 \\
$\psi_{\text {out }}[\mathrm{C} / \mathrm{m}]$ & 0.0114 & -0.0240 & -0.0122 & 0.0004 \\
$\psi$ & -93.53 & -62.92 & 37.02 & -158.61 \\
\hline
\end{tabular}

poled in the $x_{3}$ (resp. $x_{1}$ ) direction (Fig. 8). Because of the thin beam geometry and the range of interest of vibratory modes, the numerical study comes down to extensional modes along the axis $x_{1}$.

The primary and secondary sides have to be separately created by introducing 2 local coordinate systems to define the material matrices of each piezoelectric ceramic from (35) and (36). After defining the structure, the determination of the electromechanical characteristics requires a modal study. This procedure, in which the primary and secondary sections are short-circuited and open, respectively, enables the determination of the modal mass, the modal stiffness, the modal electromechanical conversion factors, and the modal transformer ratio. This method relies on the calculus for each longitudinal mode of the resonant frequency $f_{p}$, the maximum amplitude of displacement $\eta_{m}$, the kinetic energy $T$, the potential energy $U$, the charge on the primary electrode $q_{\text {in }}$, and the secondary electrical potential $V_{\text {out }}$. By knowing these different quantities, the electromechanical parameters can be obtained by the following equations:

$$
\begin{gathered}
M=\frac{2 T}{\left(2 \pi f_{p}\right)^{2} \eta_{m}^{2}}, \quad K=\frac{2 U}{\eta_{m}^{2}}, \\
\psi_{\text {in }}=m \frac{q_{\text {in }}}{\eta_{m}}, \quad \psi_{\text {out }}=\frac{C_{\text {out }} V_{\text {out }}}{\eta_{m}} .
\end{gathered}
$$

According to the previous expressions of the electromechanical parameters, the electrical characteristics (motional inductance and motional capacitance) can be extracted as follows:

$$
L_{m}=\frac{M}{\psi_{\mathrm{in}}^{2}}, \quad C_{m}=\frac{\psi_{\mathrm{in}}^{2}}{K}
$$
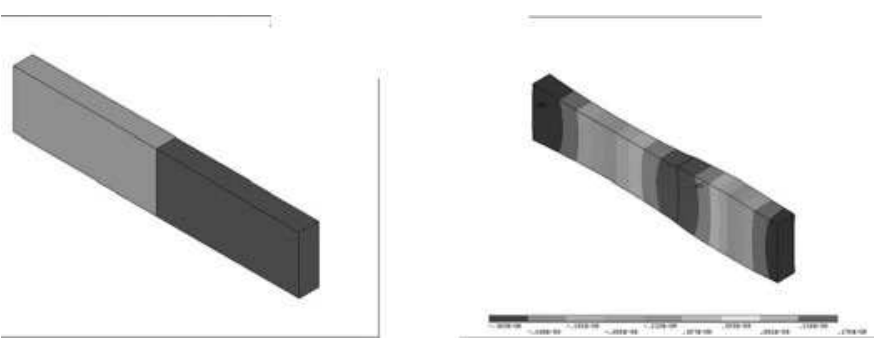

Fig. 8. 3-D deformed structure.
A modal study where the driving and receiving sections are short-circuited is also undertaken to extract the series resonant frequency $f_{s}$. The modal values are itemized in Table V.

\section{B. Validation of Analytical Models}

Figs. 9 and 10 show the numerically obtained normalized mechanical shape and electrical potential, respectively, of the first 4 modes. It appears clear that the curves, as much for the modal mechanical displacements as for the modal electrical potentials, are quite similar with the ones from nonhomogeneous Rosen-type PT model. The nodes and antinodes of modal shapes are effectively located in the left half of the structure in conformity with the nonhomogeneous model. Furthermore, the vibratory behavior defined by the modal parameters is in line with the analytical values for the $(\lambda / 2)$ - and $(\lambda)$-modes. The peculiar vibratory behavior of the $(2 \lambda)$-mode, underlined by the theoretical model, is also confirmed by the numerical study. Indeed, the very close values of series resonant frequencies $f_{s}$ and $f_{p}$ and the weak electromechanical conversion factors $\psi_{\text {in }}$ and $\psi_{\text {out }}$ can testify to this phenomenon.

As the experimental identification is obtained by admittance measurement, Fig. 11 shows the input admittance simulated by analytical and numerical models and the latter are compared with experimental results. Fig. 12 illus-

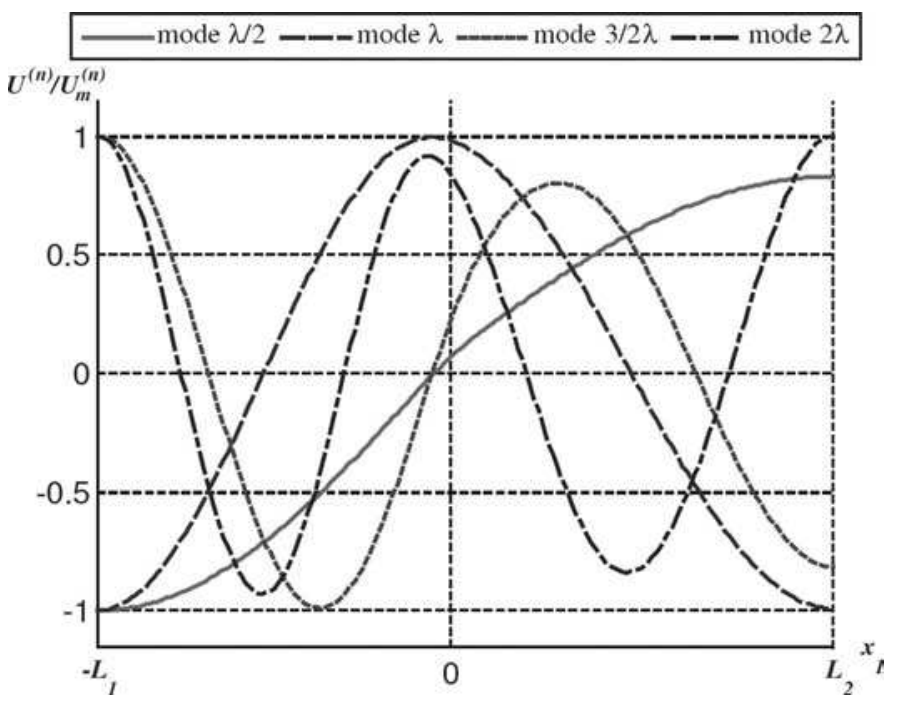

Fig. 9. Numerical normalized mechanical shape of the first 4 modes. 


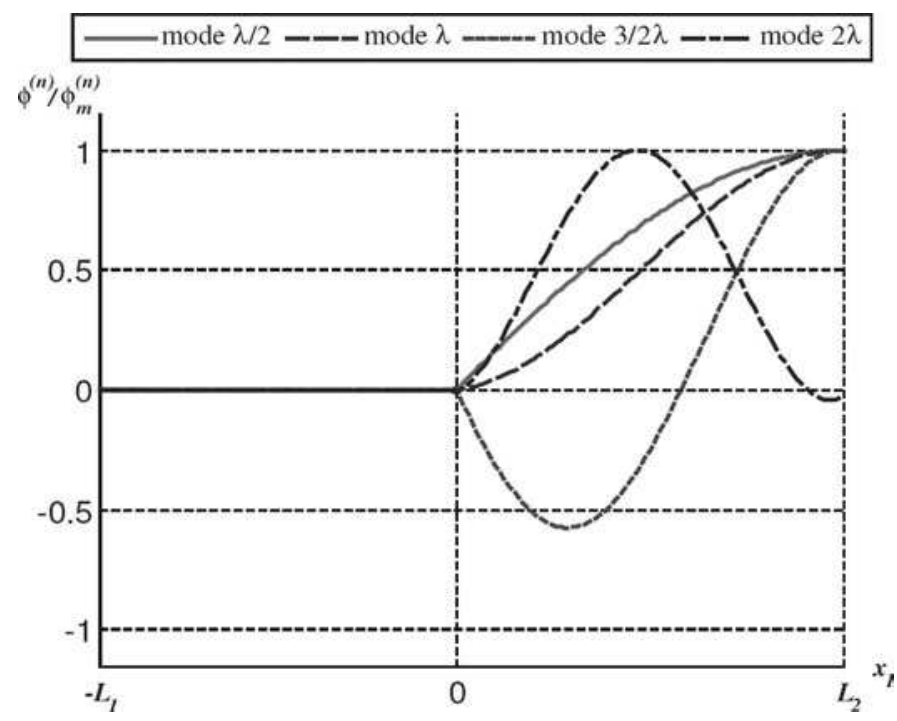

Fig. 10. Numerical normalized electrical potential of the first 4 modes.

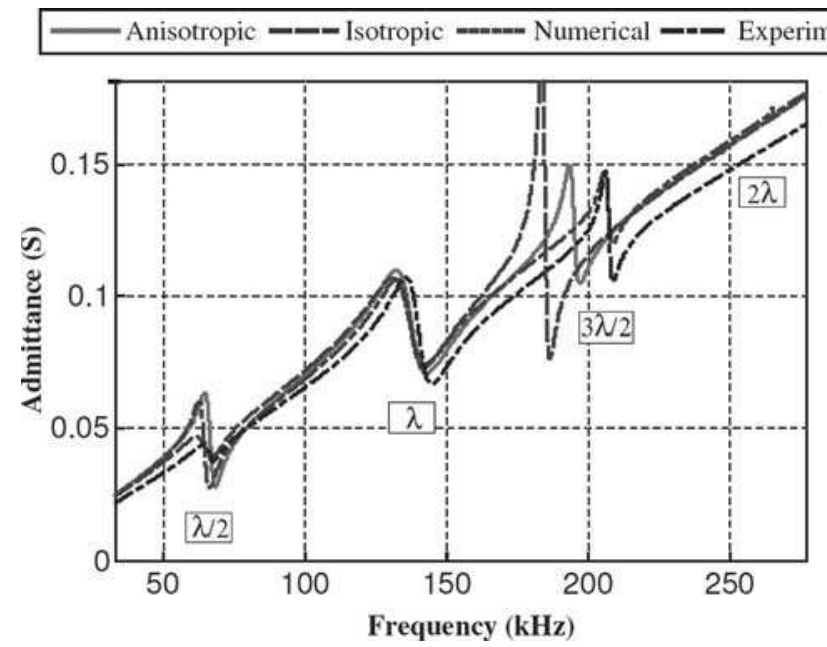

Fig. 11. Input admittance modulus of Rosen piezoelectric transformer for $500 \mathrm{k} \Omega$ load.

trates the voltage step-up ratio obtained by simulation of the equivalent multimodal electrical circuit (Fig. 5). According to these both figures, a convenient accuracy for the first $2(\lambda / 2)$ - and $(\lambda)$-modes can be noted between the different approaches. This comparison proves the validity of the assumption (66) about consideration of homogeneous medium. Then, an obvious divergence between models appears at the third longitudinal vibratory mode. If the nonhomogeneous model may be considered as acceptable, the homogeneous one presents a widely undervalued resonant frequency. From this mode, it can be considered the limit of validity of 1-D approximation for these dimensions, whereas the numerical model is still convenient. Another comment can be made about the peculiar $(2 \lambda)$-mode: if the analytical and numerical models shows its existence, the first transverse mode predominates experimentally in the vicinity of the latter and the identification of its modal

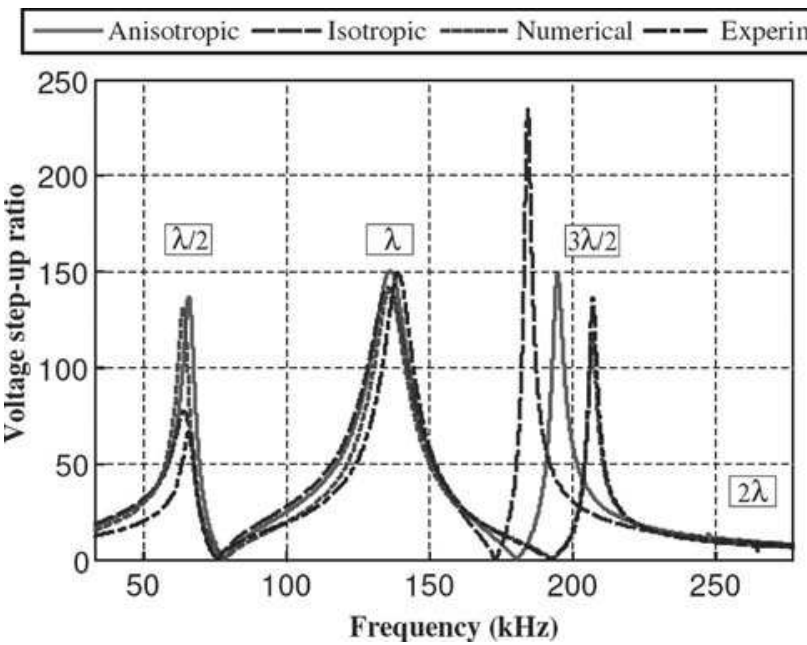

Fig. 12. Voltage step-up ratio of Rosen piezoelectric transformer for $500 \mathrm{k} \Omega$ load.

characteristics is made impossible. The frequency location of this first transverse mode can be proved by a numerical study and is also confirmed in [14].

Finally, it can be noted that amplitude accuracy of nonhomogeneous and numerical models depends on load choice. Indeed, waveform is affected by the latter, leading to displacement of the nodes and consequently the modal parameters values.

\section{CONCLusion}

A general multimodal electromechanical method of a freely vibrating PT based on Hamilton's principle was presented. The main interest of this analytical approach was to obtain a dimensioning model for inverse problem formulation to deduce the optimal dimensions. Moreover, this general method can be applied to several kinds of PT geometry with multiple driving and receiving sections, and also without imposing beforehand a vibratory mode rank. To validate the proposed model, the classic Rosen-type PT was considered and the nonhomogeneous and homogeneous PT assumptions are made. The modal characteristics, mechanical shapes, and electrical potentials were obtained and compared for both approaches. The analytical models are pertinent and their use depends only on the considered point of interest (for example: electrical potential repartition study, single-mode study, etc.). Finally, a comparison between the theoretical, numerical, and experimental results was presented and a good agreement was obtained.

\section{Appendix A}

- Frequency equation of the nonhomogeneous Rosentype PT model: 


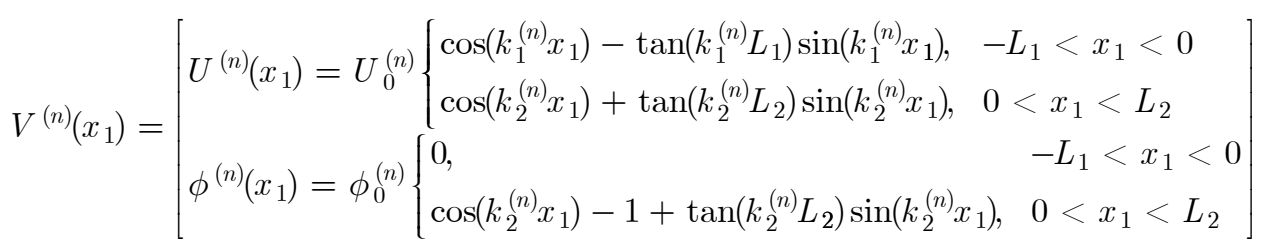

$$
\begin{gathered}
\sqrt{\frac{s_{33}^{E}\left(1-k_{33}^{2}\right)}{s_{11}^{E}}} \sin \left(k_{1}^{(n)} L_{1}\right) \cos \left(k_{2}^{(n)} L_{2}\right) \\
+\cos \left(k_{1}^{(n)} L_{1}\right) \sin \left(k_{2}^{(n)} L_{2}\right)=0
\end{gathered}
$$

- General solution of eigenvalue problem based on the nonhomogeneous Rosen-type PT model: see (A2), above.

- Normalization constant of modal mechanical shapes from the nonhomogeneous Rosen-type PT model:

$$
U_{0}^{(n)}=\frac{1}{\sqrt{\frac{1}{2} \rho w t\left[L_{1} \frac{1+\operatorname{sinc}\left(2 k_{1}^{(n)} L_{1}\right)}{\cos ^{2}\left(k_{1}^{(n)} L_{1}\right)}+L_{2} \frac{1+\operatorname{sinc}\left(2 k_{2}^{(n)} L_{2}\right)}{\cos ^{2}\left(k_{2}^{(n)} L_{2}\right)}\right]}}
$$

\section{REFERENCES}

[1] C. Rosen, "Ceramic transformers and filters," in Proc. Electronic Components Symp., 1956, pp. 205-211.

[2] S.-T. Ho, "Design of the longitudinal mode piezoelectric transformer," in 7th Int. Conf. Power Electronics and Drive Systems, Nov. 2007, pp. 1639-1644.

[3] S.-T. Ho, "Modeling and analysis on ring-type piezoelectric transformer," IEEE Trans. Ultrason. Ferroelectr. Freq. Control, vol. 54, no. 11, pp. 2376-2384, Nov. 2007.

[4] F. Pigache, F. Messine, and B. Nogarede, "Optimal design of piezoelectric transformers: A rational approach based on an analytical model and a deterministic global optimizations," IEEE Trans. Ultrason. Ferroelectr. Freq. Control, vol. 54, no. 7, pp. 1293-1302, July. 2007.

[5] H. Tiersten, "Hamilton's principle for linear piezoelectric media," in Proc. IEEE, vol. 55, no. 8, pp. 1523-1524, Aug. 1967.

[6] N.-W. Hagood and A.-J. McFarland, "Modeling of a piezoelectric rotary ultrasonic motor," IEEE Trans. Ultrason. Ferroelectr. Freq. Control, vol. 42, no. 42, pp. 210-224, Mar. 1995.

[7] M. Geradin and D. Rixen, Mechanical Vibrations Theory and Applications to Structural Dynamics, 2nd ed. New York, NY: Wiley, 1997.
[8] IEEE Standard on Piezoelectricity, ANSI/IEEE Standard 176-1987, 1988.

[9] E. Horsley, M. Foster, and D. Stone, "State-of-the-art piezoelectric transformer technology," presented at Eur. Conf. Power Electronics and Applications, 2007.

[10] J. Yang and X. Zhang, "Extensional vibration of a nonuniform piezoceramic rod and high voltage generation," Int. J. Appl. Electromagn. Mech., vol. 16, no. 12, pp. 29-42, 2002.

[11] http://www.noliac.com/Default.aspx?ID $=7781$

[12] J. F. Lopez, "Modeling and optimization of ultrasonic linear motors," Ph.D. dissertation, Ecole Polytechnique Fédérale de Lausanne, November 2006.

[13] R.-L. Lin, "Piezoelectric transformer characterization and application of electronic ballast," Ph.D. dissertation, Virginia Polytechnic Institute and State University, Nov. 2001.

[14] V. Karlash, "Electroelastic vibrations and transformation ratio of a planar piezoceramic transformer," J. Sound Vibrat., vol. 277, pp. 353-367, 2004.

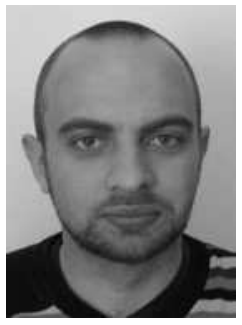

Clement Nadal was born in Rennes, France, in 1983. He received the Dipl. Ing. degree in electrical engineering from Ecole Nationale Supérieure d'Electrotechnique, d'Electronique, d'Informatique, d'Hydraulique et des Télécommunications (ENSEEIHT), Toulouse, France, and the M.S. degree in electrical engineering from the Institut $\mathrm{Na}-$ tional Polytechnique de Toulouse in 2007. He is now a Ph.D student in the Electrodynamics GREM3 research group of the LAboratoire des PLAsmas et de la Conversion d'Energie (LAPLACE), Toulouse, France.

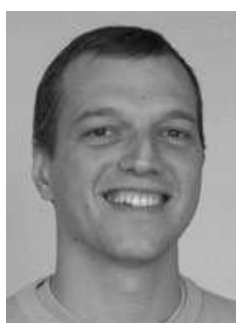

Francois Pigache was born in Auchel, France, in 1977. He received the M.S. degree in instrumentation and advanced analysis in 2001, and the Ph.D. degree in electrical engineering from the University of Sciences and Technology of Lille, France, in 2005. He joined the LAboratoire des PLAsmas et de la Conversion d'Energie (LAPLACE), Toulouse, France, as an assistant professor where his research concerns the multi-physics modeling and the optimization of piezoelectric transformers. 